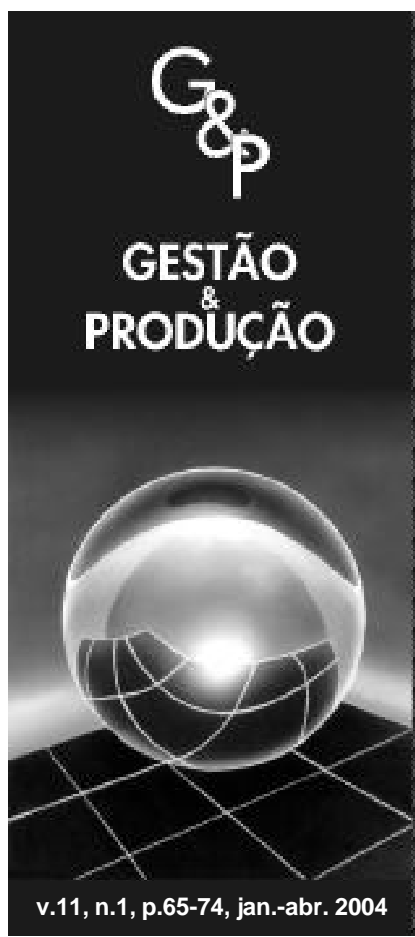

\title{
TEMPO DE IMPLANTAÇÃO DE SISTEMAS ERP: ANÁLISE DA INFLUÊNCIA DE FATORES E APLICAÇÃO DE TÉCNICAS DE GERENCIAMENTO DE PROJETOS
}

\author{
Thais Cássia Cabral Padilha \\ Oracle Corporation, e-mail: thais.padilha@oracle.com
}

Antônio Fernando Branco Costa

José Luiz Contador

Fernando Augusto Silva Marins

Faculdade de Engenharia, Campus de Guaratinguetá, UNESP, e-mails: fbranco@feg.unesp.br, jluiz@feg.unesp.br e fmarins@feg.unesp.br

Recebido em 14/8/2002

Aceito em 2/3/2004

Resumo

Freqüentemente, a implantação de sistemas ERP é complexa e demorada, requerendo, em alguns casos, três ou quatro anos. Em geral, um sistema ERP divide-se em módulos cujas implantações são feitas em vários estágios. Um problema sério é que os prazos para a implantação desses módulos são críticos e raramente são cumpridos. Esses atrasos geram insatisfação dos clientes, pois resultam em custos adicionais não previstos. A implantação de sistemas ERP depende de vários fatores, alguns dos quais têm muita influência nos prazos de implantação. Considera-se neste trabalho a técnica de planejamento de experimentos para a determinação dos principais fatores. Além disso, são usados métodos de caminhos críticos para a identificação das atividades que requerem mais investimentos para que haja redução no tempo total de implantação do projeto.

Palavras-chave: implantação de sistemas ERP, planejamento de experimentos, métodos de caminhos críticos.

\section{Introdução}

No atual cenário empresarial mundial as empresas buscam aumentar sua competitividade, seja pela redução de custos, pela melhoria do produto, agregando mais valor a ele e se diferenciando da concorrência, ou pela especialização em algum segmento de mercado. A competição tem escalas globais, assim, acontecimentos em países distantes podem trazer consequiências instantâneas para a indústria local.

Muitas empresas estão optando pelos pacotes ERP (Enterprise Resource Planning) por vários motivos, como: frustrações com sistemas incompatíveis, departamentos de tecnologia de informação que não possibilitam a integração entre esses sistemas, não consolidam as mudanças relacionadas à troca de moeda na Europa e outros que influenciam diretamente na obtenção de maior competitividade.

A sigla ERP traduzida literalmente significa "Planejamento dos Recursos da Empresa", o que pode não refletir a realidade de seus objetivos. Koch et al. (2001) afirmam: "...esqueça a parte do planejamento - ele não o faz, e esqueça os recursos, é apenas um termo de ligação. Mas lembre-se da parte da empresa. Esta é a real ambição dos sistemas ERP". Os sistemas ERP, também chamados no Brasil de Sistemas Integrados de Gestão Empresarial, controlam e fornecem suporte a todos os processos operacionais, produtivos, administrativos e comerciais da empresa.

Todas as transações realizadas pela empresa devem ser registradas, a fim de que as consultas extraídas do sistema possam refletir a realidade. O ERP é um sistema integrado que possibilita um fluxo de informações único, contínuo e consistente por toda a empresa, sob uma única base de dados. É um instrumento para a melhoria de processos como a produção, compras ou distribuição, informações on-line e em tempo real. Em suma, o sistema permite visualizar as transações efetuadas pela empresa desenhando um amplo cenário de seus negócios. 
O cumprimento de prazos e orçamentos por parte de empresas fornecedoras de sistemas ERP tem sido um ponto crítico na implantação desse tipo de programa. De acordo com Gomes \& Vanalle (2001), algumas das causas para o não cumprimento de cronogramas podem ser: a resistência das pessoas da organização envolvidas no projeto, limitações inerentes ao próprio ERP escolhido e a dificuldade de integrar o ERP com diversos sistemas existentes dentro da empresa, entre outras. Assim, uma fornecedora de sistemas ERP deve se preocupar com a determinação dos fatores que influenciam seu tempo de implantação. Com a determinação desses fatores é possível controlar ou ao menos estipular, com menor margem de erro, a duração de um projeto.

Nesse contexto, observa-se que há grande oportunidade para o uso das técnicas de planejamento e análise de experimentos como ferramentas de auxílio na obtenção de prazos a serem cumpridos pela empresa fornecedora do sistema.

Com as análises realizadas neste estudo busca-se melhorar a qualidade do serviço prestado pela empresa fornecedora de aplicativos de sistemas ERP, na medida em que facilitam o cumprimento de prazos e cronogramas preestabelecidos. Segundo Campos (1995), o cumprimento de prazos é um dos principais requisitos para a qualidade assegurada. Com a análise de experimentos e a utilização de ferramentas estatísticas, verifica-se que os fatores estudados, como o ano de implantação do ERP e o porte da empresa/cliente, possuem grande influência no tempo total de implantação desses sistemas. Dessa forma, pode-se procurar segmentar os clientes em grupos distintos de acordo com os níveis dos fatores estudados.

Este trabalho apresenta um estudo de caso em que técnicas de Planejamento de Experimentos foram utilizadas a fim de melhorar a qualidade de serviço de uma empresa de consultoria em relação à determinação do tempo de execução dos projetos vinculados a Sistemas ERP. Além disso, foi utilizada a ferramenta Técnica de Avaliação e Revisão de Programas (PERT) com o objetivo de avaliar cada atividade do projeto e definir as atividades-chave (ou críticas) em que devem ser alocados mais recursos para garantir (reduzir) a duração programada. Mais detalhes sobre PERT e suas aplicações podem ser encontrados em Contador (1997).

\section{Técnicas de planejamento e análise de experimentos}

Em experimentos industriais é comum estudar o efeito de um ou mais fatores na variável "resposta". Exemplo: qual a influência do catalisador no tempo de uma reação química? Observa-se que o número de experimentos cresce com o número de fatores, pois na análise é preciso considerar todas as possíveis combinações dos níveis dos fatores do experimento. Exemplo: sejam os fatores pressão e temperatura e deseja-se analisar a influência deles na velocidade de uma reação química. Se forem definidas três pressões e três temperaturas, um experimento completo demandará a estimativa de nove velocidades da reação química, uma para cada combinação de valores de pressão e de temperatura.

Quando o número de fatores é grande, recorre-se aos experimentos fatoriais fracionados, em que é realizada apenas uma parte, bem definida, do experimento completo (Montgomery, 2001).

No estudo dos resultados dos experimentos busca-se identificar o efeito produzido na variável resposta analisando a variação dos níveis dos fatores de controle do experimento. Os efeitos são classificados como principal, que representa a alteração média da variável resposta resultante da mudança de nível de um fator mantendo-se os outros fatores fixos, ou de interação, quando a variação da resposta é decorrente da mudança combinada dos níveis de dois ou mais fatores.

Para a análise dos resultados podem ser utilizados procedimentos gráficos. Entretanto, Montgomery (2001) afirma que o uso de técnicas estatísticas para analisar os resultados é mais vantajoso e sugere, em especial, a análise de variância (ANOVA). Com a análise de variância é possível avaliar, com a segurança estatística desejada, se os efeitos são significativamente diferentes de zero e, com isso, selecionar os fatores que influenciam na variável resposta.

\subsection{Seleção dos fatores de controle e os níveis do processo de implantação de sistemas}

O objetivo do experimento foi verificar os fatores que influenciam o tempo de implantação de módulos de sistemas ERP. Dessa forma, definiu-se como variável resposta do experimento o tempo total de implantação do conjunto de módulos. As principais causas de atraso na implantação, segundo Gomes \& Vanalle (2001), são:

1. a resistência por parte das pessoas envolvidas no projeto;

2. rotatividade dos funcionários que foram treinados no novo sistema ou que dominam as atividades da empresa;

3. qualidade da equipe de consultoria contratada;

4. limitações inerentes ao próprio produto ERP escolhido;

5. dificuldade de integrar o ERP com outros sistemas existentes dentro da empresa ou corporação;

6. porte da empresa onde o sistema será implantado.

$\mathrm{Na}$ empresa estudada foram escolhidos funcionários dos setores envolvidos para participarem do estudo em questão. As reuniões foram realizadas semanalmente para discutir o experimento e os procedimentos para a obtenção dos dados. Os fatores 1 e 2, dependentes essencialmente do desempenho das pessoas envolvidas, são de difícil mensuração. Acredita-se, ainda, que o comportamento inicial dessas pessoas seja semelhante em todas as empresas, não sendo necessário avaliar esses fatores. 
O fator 3, qualidade da equipe de consultoria, tem grande importância no tempo de implantação do sistema. A empresa analisada já realizou estudos para determinar a influência do conhecimento da consultoria sobre o tempo de implantação. As equipes são, atualmente, divididas em cinco níveis de conhecimento. O tempo de que cada uma dessas equipes necessita para desenvolver seu trabalho é inversamente proporcional a seu patamar de conhecimento. Dessa forma, para realizar este estudo foi fixado o nível 3 para todas as empresas clientes envolvidas.

O fator 4 foi excluído dos fatores de controle, uma vez que o sistema implantado é sempre o mesmo e, portanto, a dificuldade com suas limitações já são conhecidas e invariáveis em qualquer empresa estudada. De maneira análoga, o fator 5 foi controlado, pois será analisada somente a implantação de módulos e não o sistema ERP completo, tornando necessária a comunicação desses módulos com outros sistemas da empresa.

De acordo com o conhecimento já adquirido pela empresa de consultoria em estudo, dois fatores deveriam ser analisados: o porte da empresa na qual o sistema será implantado (denominada empresa-cliente) e o ano de implantação do sistema. Ainda com base no conhecimento empírico dos envolvidos no processo, foram definidos os níveis de cada fator. Para o fator ano de implantação do sistema ERP, acredita-se que, a partir de 1999, a consultoria e o próprio sistema tenham amadurecido e facilitado o processo de implantação. Dessa forma, apresenta dois níveis: antes e a partir de 1999.

Para o fator porte da empresa-cliente foram definidos três níveis a partir de seu faturamento anual. As empresas foram classificadas em pequena, média e grande, com faturamento de até $R$ \$ 300 milhões, entre $R$ \$ 301 e $R$ \$ 999 milhões e acima de $\mathrm{R} \$ 1$ bilhão, respectivamente. É importante ressaltar que essa divisão somente classifica as empresas para esta análise, já que empresas com esse nível de faturamento são consideradas de médio porte para cima. A hipótese a ser confirmada com a análise desse fator é que o volume de informações para configurar o aplicativo é proporcional ao porte da empresa, influenciando no tempo de implantação que é a variável resposta do experimento.

Como um dos fatores a analisar é o ano de implantação, foram utilizados dados históricos da empresa. Para obter o volume de informação necessária, alguns procedimentos foram realizados. Determinou-se que a análise incluiria somente o tempo de implantação de alguns pacotes comerciais compostos por 10 módulos cada. Dessa forma, todas as empresas-clientes consideradas implantaram os mesmos módulos (fator de controle 5). Os módulos analisados foram o financeiro (livro fiscal, contas a pagar e contas a receber), o de manufatura (MRP, listas de materiais, programação, controle, capacidade, qualidade) e o de compras. $\mathrm{O}$ fator 3 , citado anteriormente, também foi controlado, sendo utilizados apenas os dados em que a consultoria apresentava nível três.

\section{Aplicação das técnicas de planejamento e análises de experimentos no estudo do tempo de implantação de módulos de sistemas ERP}

Estabelecidos os fatores e seus níveis, partiu-se para o levantamento de dados.

Formulação das hipóteses a serem testadas:

- $\mathrm{H}_{0 \mathrm{~A}}$ : o fator ano de implantação não interfere na média do tempo de implantação;

- $\mathrm{H}_{\mathrm{OP}}$ : o fator porte da empresa-cliente não interfere na média dos tempos de implantação;

- $\mathrm{H}_{0 \mathrm{I}}$ : não há interação entre fatores;

- $\mathrm{H}_{1 \mathrm{~A}}$ : o fator ano de implantação interfere na média do tempo de implantação;

- $\mathrm{H}_{\mathrm{IP}}$ : o fator porte da empresa-cliente interfere na média do tempo de implantação;

- $\mathrm{H}_{11}$ : há interação entre os fatores testados.

Alternativamente, pode-se dizer que o seguinte modelo será testado:

$\mathrm{Y}_{\mathrm{ij}}=\mu+\tau_{\mathrm{i}}+\beta_{\mathrm{j}}+\tau_{\mathrm{i}} \beta_{\mathrm{j}}+\xi_{\mathrm{ij}}, \operatorname{com} \mathrm{i}=1,2 \mathrm{e} \mathrm{j}=1,2,3$,

em que $Y_{\text {ij }}$ representa o tempo de implantação nas condições em que o fator ano de implantação se encontra no nível i e o fator porte da empresa-cliente está no nível j, $\mu$ é a média global, $\tau_{\mathrm{i}}$ é o efeito do i-ésimo nível do fator ano de implantação, $\beta_{\mathrm{j}}$ é o efeito do j-ésimo nível do fator porte da empresa-cliente, $\tau \tau_{\mathrm{i}}$ é o efeito da interação entre o i-ésimo nível do fator ano de implantação e o j-ésimo nível do fator porte da empresa-cliente e $\xi_{\mathrm{ij}}$ é o erro aleatório usual.

A Tabela 1 apresenta valores da variável resposta (tempo de implantação) para os diferentes níveis dos fatores. O Quadro 1 apresenta os resultados da análise de variância (ANOVA). Comparando-se o $\mathrm{F}$ tabelado para $1 \%$ com o $\mathrm{F}_{\mathrm{o}}$ calculado a partir da amostra, verifica-se que ambos os fatores, ano de implantação e porte da empresa, afetam o tempo de implantação. Entretanto, observa-se que não há interação entre os fatores. Portanto, foram rejeitadas as hipóteses $\mathrm{H}_{0 \mathrm{~A}} \mathrm{e} \mathrm{H}_{\mathrm{OP}}$, e aceita a hipótese $\mathrm{H}_{0}$.

Realmente houve amadurecimento do aplicativo e da empresa após 1999, reduzindo o tempo de implantação e mostrando que o nível de faturamento da empresa interfere na duração do projeto. Como foram confirmadas as hipóteses $\mathrm{H}_{1 \mathrm{~A}}$ e $\mathrm{H}_{1 \mathrm{P}}$, é necessário saber se os tempos médios de implantação em empresas de pequeno e médio portes, ou de médio e grande portes, e assim por diante, diferem significativamente. Para realizar esse teste foi utilizado o método de Tukey (Costa Neto, 1997). 
Tabela 1 - Valores da variável resposta (semanas) para as combinações dos níveis dos fatores.

\begin{tabular}{|c|c|c|c|c|c|c|c|c|c|c|}
\hline \multirow{2}{*}{ Ano } & \multicolumn{9}{|c|}{ Tipo de empresa } & \multirow{3}{*}{$\frac{\text { Soma }}{323}$} \\
\hline & \multicolumn{3}{|c|}{ Pequena } & \multicolumn{3}{|c|}{ Média } & \multicolumn{3}{|c|}{ Grande } & \\
\hline \multirow{5}{*}{ Antes de 99} & 6 & 7 & 71 & 10 & 11 & 105 & 15 & 11 & 147 & \\
\hline & 8 & 10 & & 12 & 11 & & 12 & 14 & & \\
\hline & 5 & 4 & & 10 & 12 & & 13 & 18 & & \\
\hline & 8 & 7 & & 10 & 10 & & 12 & 18 & & \\
\hline & 9 & 7 & & 9 & 10 & & 18 & 16 & & \\
\hline \multirow{6}{*}{ Depois de 99} & 3 & 7 & 45,5 & 7 & 5 & 63,5 & 10 & 8 & 93 & 202 \\
\hline & 5 & 4 & & 10 & 8 & & 12 & 8 & & \\
\hline & 6 & 3 & & 8 & 5 & & 12 & 8 & & \\
\hline & 4,5 & 4 & & 6 & 4 & & 7 & 10 & & \\
\hline & 4 & 5 & & 6 & 4,5 & & 11 & 7 & & \\
\hline & & & 116,5 & & & & & & 240 & 525 \\
\hline
\end{tabular}

\subsection{Comparação entre as médias - método Tukey}

Como há diferença entre os níveis do fator tamanho da empresa-cliente, é necessário realizar um teste estatístico para identificar entre quais níveis desse fator há diferenças. Neste trabalho optou-se pelo Teste de Tukey (Montgomery, 2001).

Formulação das hipóteses:

- $H_{\mathrm{o}}$ : Não há diferença entre os níveis i e j do fator porte da empresa, $i \neq j \in\{$ pequeno, médio, grande $\}$;

- $\mathrm{H}_{1}$ : Há diferença entre esses níveis.

O Quadro 1 contém as médias dos tempos de implantação para cada nível do fator tamanho da empresa, bem como as diferenças, em módulo, entre essas médias para cada dois níveis do fator tamanho da empresa. Todas as diferenças excederam a 1,9 semana, que é o valor obtido pelo método de Tukey para um risco $\alpha=1 \%$. Conclui-se, assim, que os tempos médios de implantação nas empresas pequenas é menor que nas médias, que, por sua vez, é menor que em empresas grandes.

\section{Considerações acerca da análise de fatores}

Com a análise dos fatores que influenciam o tempo de implantação de módulos de sistemas ERP, a empresa fornecedora do aplicativo possui agora mais informações para elaborar um cronograma preciso, garantindo, assim, o prazo definido no projeto. Com o conhecimento de que o tempo de execução de um projeto depende do nível de faturamento da empresa, pode-se estimar melhor sua duração.

A influência dos fatores analisados é vista por meio do feeling dos gerentes de projeto e, dessa forma, não é consi- derada na programação de venda do sistema. O departamento de vendas normalmente estipula um prazo curto para o projeto com o objetivo de satisfazer o cliente mais rapidamente, mas sem conhecer realmente sua duração. Com a constatação sobre a diferença de tempo de projeto para diferentes portes de empresa, o departamento de vendas pode, agora, estipular prazos diferenciados para cada cliente.

Para garantir não somente maior confiabilidade na determinação dos prazos, mas também que os mesmos sejam efetivamente cumpridos, procurou-se analisar o projeto com mais detalhes. Nessa análise, foi utilizada a ferramenta PERT (Contador, 1997), com o objetivo de avaliar cada atividade do projeto e definir as atividades críticas, nas quais devem ser utilizados mais recursos para garantir a duração programada. Esta análise é apresentada na seção seguinte.

\section{Gerenciamento do projeto de implantação de sistemas ERP}

\subsection{Considerações sobre gerenciamento de projetos}

Freqüentemente, o gerenciamento de projetos acarreta várias questões conflitantes, como: não há tempo para executar a tarefa, o trabalho é muito complexo ou o orçamento não é adequado. Para essas situações, Strauss (1997) recomenda entender e considerar as três dimensões gerais do gerenciamento de projeto: tempo, tarefa e recursos. $\mathrm{O}$ autor destaca ainda que "sem um entendimento de como estes três fatores se inter-relacionam, o gerente pode facilmente entrar em modo reativo, constantemente respondendo à crise do momento".

Esses três fatores constantemente interagem em um projeto mudando a prioridade e variando a importância conforme o projeto avança. Entender como esses fatores interagem fornece uma perspectiva objetiva do processo de desenvolvimento. Portanto, essa é uma tarefa típica do 
gerente de projeto: gerenciar esses fatores e tomar as decisões. É importante perceber que, em projetos distribuídos, qualquer decisão tomada sobre determinado projeto desencadeia alterações a serem feitas nos demais projetos correlacionados. Assim, os gerentes devem estar cientes de toda e qualquer ação efetuada. As três dimensões e suas interações podem ser visualizadas na Figura 1. Cada uma das dimensões pode ser definida e exemplificada da seguinte maneira:

- Tempo: o tempo requerido refere-se ao cronograma, especialmente ao deadline para a tarefa (data final). Essa data depende da natureza da tarefa (projeto) e da disponibilidade de recursos. A análise de tempo (ou prazos) preocupa-se com a decomposição do projeto em atividades (ou tarefas) e com a interligação destas. O principal produto da análise de tempo é o sequienciamento das atividades com as datas de início e de término e as folgas de cada atividade.

- Tarefa: refere-se ao que está sendo desenvolvido. É o escopo do trabalho a ser realizado: a grandeza e a complexidade da aplicação final. Ou seja, consiste na especificação dos requisitos, no projeto funcional, etc. A definição do produto final, segundo Strauss (1997), determinará o número de pessoas necessárias para produzir a aplicação, as habilidades das pessoas, o tipo de equipamento e quanto tempo levará para completar o projeto.

- Recursos: a análise de recursos se preocupa em adequar as datas produzidas pela análise de tempo à disponibilidade de recursos. Nessa análise utilizam-se as folgas das atividades, que nem sempre são suficientemente extensas para a completa adequação entre datas e recursos. O produto é uma nova programação das atividades em que a utilização de recursos está em níveis compatíveis com a disponibilidade. Os custos representam uma análise particular dos recursos e, basicamente, se referem a quanto dinheiro está disponível para ser gasto no projeto e como esse dinheiro é aplicado em pessoas, material e equipamento.

Dessa forma, as ferramentas de gerenciamento de projetos atuam no âmbito desses três fatores, permitindo gerenciar e planejar cada um deles, bem como a interação entre os mesmos. E no escopo de projetos distribuídos, permite gerenciar os fatores quando ocorrerem modificações no planejamento inicial de algum dos projetos. Em relação ao uso de técnicas quantitativas para a gerência de projetos, pode-se relacionar, segundo Prado (1998):

- Tempo - as principais técnicas disponíveis são o diagrama de barras (Gráfico de Gantt) e o diagrama PERT/CPM. $\mathrm{O}$ uso de um ou outro depende da complexidade do projeto.

- Recursos - no caso de redistribuição de recursos, usase a simulação da execução do projeto, quando se modificam as folgas das atividades para não ultrapassar as disponibilidades dos recursos. Em pequenos projetos, tal estudo pode ser executado manualmente. Em médios e grandes projetos, o computador é fundamental para obter uma otimização na utilização de recursos.

Quadro 1 - Quadro de análise de variância.

\begin{tabular}{lccccccc}
\hline & Fonte & SQ & GL & QM & Fo & Ftab 5\% & Ftab 1\% \\
\hline Ano & 1 & 244,02 & 1 & 244,02 & 61,62 & 4,08 & 7,31 \\
$\begin{array}{l}\text { Tamanho da } \\
\text { empresa }\end{array}$ & 2 & 384,48 & 2 & 192,24 & 48,55 & 3,23 & 5,18 \\
Interação & 12 & 20,4 & 2 & 10,2 & 2,56 & 3,68 & 6,36 \\
\hline & Erro & 213,85 & 54 & 3,96 & & & \\
& Total & 862,75 & 59 & & & & \\
& & & & & & & \\
\end{tabular}

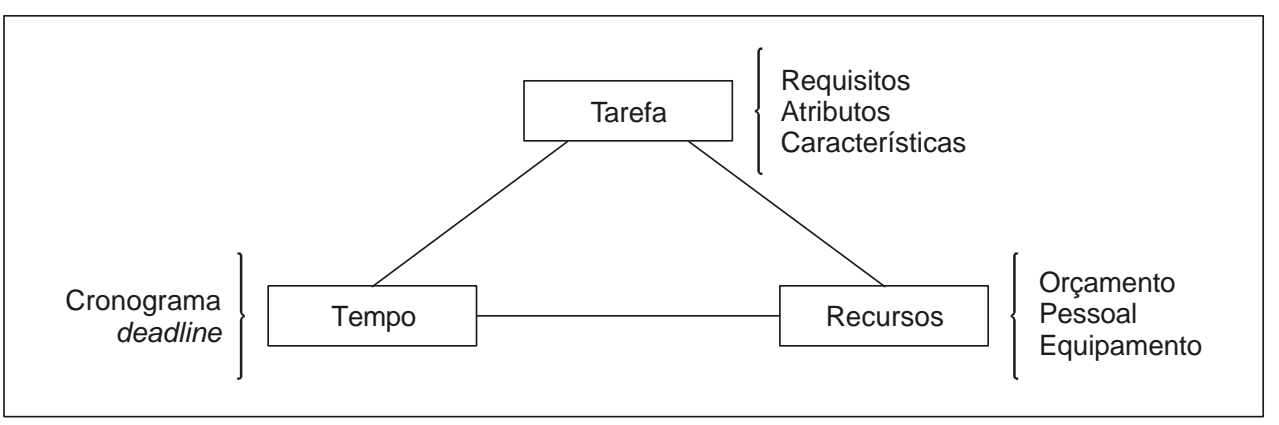

Figura 1 - Dimensões do gerenciamento de projeto. Fonte: Strauss, 1997, p. 350. 
- Custos - as técnicas mais utilizadas são os estudos orçamentários e o acompanhamento contábil. Os estudos orçamentários são baseados na decomposição do projeto em partes menores, nas quais se faz a previsão de custos. Entre as técnicas disponíveis, a mais sofisticada é o sistema earned value, que permite ligar uma estrutura de custos à rede de atividades.

Nessa etapa, a preocupação se refere ao tempo de implantação de sistemas ERP, e o primeiro passo para o planejamento de projetos é a definição das atividades do projeto e a relação de dependência entre elas.

\subsection{Seleção das atividades envolvidas no projeto e construção de rede de PERT}

A implantação de um sistema ERP obedece a uma metodologia-padrão desenvolvida para cada empresa fornecedora. Para a empresa de consultoria estudada, durante o planejamento do projeto cada fase pode ser considerada como uma atividade. A duração das atividades pode ser definida a partir das estimativas otimista $\left(\mathrm{T}_{\mathrm{o}}\right)$, pessimista $\left(\mathrm{T}_{\mathrm{p}}\right)$ e mais provável $\left(\mathrm{T}_{\mathrm{mp}}\right)$ ajustadas à distribuição beta de probabilidades, o que permite, então, encontrar a duração média e o desvio-padrão da duração de cada atividade. Para o presente trabalho, considerou-se como projeto a ser analisado a implantação dos módulos de sistemas ERP em empresas consideradas de grande porte. Uma aplicação abordará apenas as grandes atividades do projeto, em uma análise mais agregada possível, adequada, portanto, ao primeiro nível hierárquico do planejamento. O Quadro 2 mostra as principais atividades necessárias para o desenvolvimento e execução do projeto segundo a metodologia AIM (Application Implementation Method) utilizada pela empresa de consultoria estudada. No Anexo 1 apresentam-se mais detalhes sobre cada uma das atividades do projeto de implementação de aplicativos citados no Quadro 2.

A Tabela 2 apresenta as estimativas de tempo otimista $\left(\mathrm{T}_{\mathrm{o}}\right)$, pessimista $\left(\mathrm{T}_{\mathrm{p}}\right)$ e mais provável $\left(\mathrm{T}_{\mathrm{mp}}\right)$ de cada atividade, assim como suas antecessoras imediatas.

A duração média de cada atividade e seu desviopadrão, que constam na Tabela 2, foram calculados pelas fórmulas:

$$
\begin{aligned}
& \mathrm{T}_{\mathrm{ij}}=\left[\left(\mathrm{t}_{\mathrm{o}}\right)_{\mathrm{ij}}+4(\mathrm{tmp})_{\mathrm{ij}}+\left(\mathrm{t}_{\mathrm{p}}\right)_{\mathrm{ij}} / 6 ;\right. \\
& \left\|\mathrm{T}_{\mathrm{ij}}\right\|=\text { é o inteiro mais próximo de } \mathrm{T}_{\mathrm{ij}} ; \\
& \mathrm{S}_{\mathrm{i}, \mathrm{j}}^{2}=\left[(\mathrm{tp})_{\mathrm{ij}}-\left(\mathrm{t}_{\mathrm{o}}\right)_{\mathrm{ij}}\right]^{2} / 36
\end{aligned}
$$

Pelas definições anteriores obteve-se a rede de eventos (atividades no arco) do projeto que está na Figura 2.

Quadro 2 - Cálculo das diferenças entre níveis.

\begin{tabular}{lcc}
\hline \multicolumn{1}{c}{ Porte da empresa } & Médias & Cálculos das diferenças \\
\hline Pequena (P) & $\bar{X}_{P}=5,83$ & $\left|\bar{X}_{P}-\bar{X}_{M}\right|=2,62$ \\
Média (M) & $\bar{X}_{M}=8,45$ & $\left|\bar{X}_{P}-\bar{X}_{G}\right|=6,17$ \\
Grande (G) & $\bar{X}_{G}=12,0$ & $\left|\bar{X}_{M}-\bar{X}_{G}\right|=3,55$ \\
\hline
\end{tabular}

Tabela 2 - Duração (semanas) das atividades e relacionamentos.

\begin{tabular}{cccccccc}
\hline \multicolumn{2}{c}{ Atividades } & & & & & \\
\hline Atividade & Antecessora imediata & $\mathbf{T}_{\mathbf{0}}$ & $\mathbf{T}_{\mathbf{p}}$ & $\mathbf{T}_{\mathbf{m p}}$ & $\mathbf{T}_{\mathbf{i j}}$ & $\mathbf{S}_{\mathbf{i}, \mathbf{j}}$ & $\| \mathbf{T}_{\mathbf{i j}} \boldsymbol{\|}$ \\
\hline A & & 3 & 6 & 4 & 4,17 & 0,25 & 4 \\
B & & 6 & 12 & 10 & 9,67 & 1 & 10 \\
C & B & 4 & 8 & 6 & 6 & 0,44 & 6 \\
D & A,C & 8 & 16 & 12 & 12 & 1,78 & 12 \\
E & A,C & 4 & 10 & 6 & 6,33 & 1 & 6 \\
F & D,E & 3 & 6 & 4 & 4,17 & 0,25 & 4 \\
\hline TOTAL & & & & & & 42 \\
\hline
\end{tabular}


Quadro 3 - Atividades do projeto de implementação de aplicativos.

\begin{tabular}{cc}
\hline Atividades & Descrição \\
\hline A & Definição dos processos \\
B & Análise operacional \\
C & Desenho da solução \\
D & Construção \\
E & Transição \\
F & Produção \\
\hline
\end{tabular}

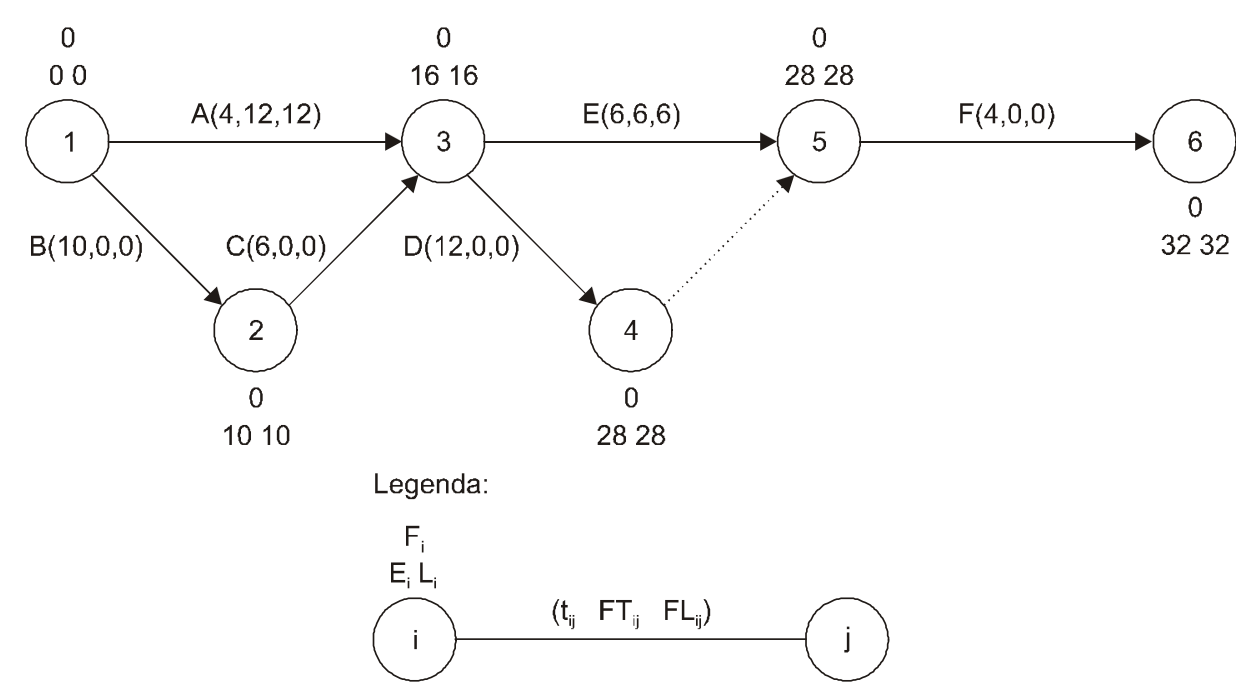

Figura 2 - Rede PERT do projeto de implantação de sistemas ERP. Legenda: $\mathrm{F}_{\mathrm{i}}$ é a folga do evento i, $\mathrm{E}_{\mathrm{i}}$ é a sua data mais cedo, $L_{i}$, sua data mais tarde, $t_{i j}$, a duração da atividade $i j, F_{i j}$, sua folga total e $F_{i j}$, sua folga livre.

\subsection{Redução da duração do projeto}

O prazo de execução de um projeto é, em muitas circunstâncias, um fator decisivo para sua viabilização, pois, como citado ao longo do trabalho, garante maior competitividade diante das concorrentes. Segundo Contador (1999), os trabalhos sobre gerenciamento de projetos utilizando a técnica PERT pouco exploram a análise estratégica do projeto, ou seja, a análise e a avaliação do projeto realizadas com o objetivo de reduzir sua duração sobre a programação originada da rede PERT (admitindo a duração média das atividades).

As análises têm se limitado à avaliação da probabilidade de cumprir prazos de conclusão enfocando apenas o caminho crítico do projeto, ou a programação das atividades para cumprir certo objetivo (como nivelar recursos). A técnica PERT para planejamento e controle de projetos é uma ferra- menta forte que permite análises estratégicas interessantes, como definir decisões para garantir maiores chances de sucesso na redução do prazo de conclusão do projeto.

Com esse objetivo, Contador (1999) desenvolveu o algoritmo do mínimo corte, que fornece um método para escolher as atividades que devem ter sua duração alterada a fim de acelerar com maior chance de sucesso a execução do projeto. A aplicação do método exige a identificação de todos os caminhos da atividade que, a partir do tempo esperado de suas tarefas, apresentam duração acima do prazo desejado de conclusão do projeto. Para tanto, Contador (2000) apresentou o algoritmo da folga mínima para determinação do caminho K-crítico de projetos. A aplicação desse algoritmo exige o conhecimento da folga total das atividades do projeto, o que pode ser facilmente obtido pela técnica PERT. Surge a opção em utilizar essa ferramenta, embora seja de um procedimento bastante antigo. 


\subsection{Algoritmo da folga mínima para determinação do caminho K-crítico de projetos}

O algoritmo da folga mínima, desenvolvido por Contador (2000) para determinar os caminhos K-críticos em uma rede PERT, baseia-se na constatação de que a folga total de uma atividade é definida pelo caminho mais longo que passa por essa atividade. Quando $\mathrm{K}=1$, tem(têm)-se o(s) caminho(s) mais longo(s) do projeto (originalmente chamados de caminhos ou caminhos críticos); para $\mathrm{K}=2$, tem(têm)-se o(s) segundo(s) caminho(s) mais longo(s) do projeto, e assim sucessivamente.

O algoritmo baseia-se na constatação de que, se houver uma atividade A com, por exemplo, a segunda menor folga entre todas as atividades do projeto, então existirá um ou mais caminhos no projeto com a segunda maior duração que passam necessariamente por essa atividade. Assim, ela pode ser escolhida como uma "semente" para a identificação desse ou desses caminhos. Aplicando-se um processo forward e backward a partir da atividade semente, escolhendo sempre atividades com a menor folga, identifica-se o segundo caminho mais longo do projeto.

Por exemplo, para realizar o projeto com duração de 24 u. t. Aplicando esse algoritmo ao projeto em questão, identificam-se os caminhos formados pelos conjuntos de atividades $\{\mathrm{B}, \mathrm{C}, \mathrm{D}, \mathrm{F}\}$ e $\{\mathrm{B}, \mathrm{C}, \mathrm{E}, \mathrm{F}\}$, com durações de 32 e 26 u. t., respectivamente, que devem, portanto, ser acelerados.

\subsection{Algoritmo do mínimo corte}

Os caminhos do projeto em questão formados pelas atividades $\{\mathrm{B}, \mathrm{C}, \mathrm{D}, \mathrm{F}\}$ e $\{\mathrm{B}, \mathrm{C}, \mathrm{E}, \mathrm{F}\}$ precisam ser acelerados. Para aplicar o algoritmo do mínimo corte deve-se classificar as atividades desses caminhos em dois grupos: (a) grupo das atividades que, segundo a avaliação gerencial e por meio de ações identificáveis, possuem boa chance de funcionar com duração menor; (b) demais atividades.

Cria-se uma rede de fluxo composta apenas pelas atividades dos caminhos a serem acelerados. Atribui-se aos arcos dessa rede limitantes inferior e superior para o fluxo, definidos de forma apropriada para, pela identificação do mínimo corte, determinar o conjunto apropriado de atividades que, uma vez aceleradas, reduzem a duração de todos os caminhos da rede de fluxo.

Essas atividades são aceleradas de uma unidade de tempo. Repete-se esse procedimento até que a duração desejada seja atingida. O conceito de mínimo corte é utilizado para localizar o conjunto formado pelo menor número de atividades que, ao terem sua duração alterada, aceleram todos os caminhos do projeto com duração superior a seu prazo de conclusão desejado. O objetivo é deslocar o menor número de atividades de seu tempo esperado. Os limitantes do fluxo em cada arco são determinados da seguinte forma:
- (a) para atividades (arcos) do grupo b: $[0, \infty]$

- (b) para atividades do grupo a: $\left[0, \mathrm{~N}^{\mathrm{k}}-\mathrm{I}^{\mathrm{k}}\right]$, em que $\mathrm{N}^{\mathrm{k}}$ é o número de caminhos da rede de fluxo (que necessitam ser acelerados) na iteração $\mathrm{k}$ e $\mathrm{I}^{\mathrm{k}}$ é o número de caminhos da rede de fluxo da iteração $\mathrm{k}$ aos quais $a$ atividade pertence.

Assim, se uma atividade não pode ser acelerada, ou seja, pertence ao grupo (b), ela jamais estará presente no corte mínimo e, portanto, nunca será acelerada. Por outro lado, se uma atividade pertencente ao grupo (a) estiver presente nos $\mathrm{N}$ caminhos da rede de fluxo, ela será necessariamente escolhida.

A Tabela 3 fornece o resultado da aplicação do algoritmo do mínimo corte para as oito iterações que levam à solução final. Inicialmente, a rede de fluxo é composta pelos caminhos (B, C, D, F) e (B, C, E, F) e, com exceção da atividade F, todas as demais foram consideradas possíveis de aceleração (grupo a). Na iteração $\mathrm{k}=4$, a atividade $\mathrm{B}$ foi excluída do grupo a (considerada imprópria para ser acelerada), ocorrendo o mesmo com a atividade $C$ na iteração $k=6$. Na iteração $\mathrm{k}=2$, o caminho (B, C, E, F) atingiu a duração desejada. Assim, a rede de fluxo passa a ser composta apenas pelo caminho (B, C, D, F). Na iteração $\mathrm{k}=8$, a duração de todos os caminhos atende ao prazo desejado e a duração resultante das atividades fornece a solução.

\section{Comentários e considerações finais}

A aplicação dos conceitos estatísticos no planejamento de experimentos se iniciou com Fisher (1966), que elaborou uma série de experimentos para seleção das melhores variedades de sementes. Por intermédio de Taguchi (1989), as idéias de Fisher passaram a ser aplicadas em experimentos industriais e, em seguida, a todo tipo de experimentos. Podese citar, por exemplo, o uso desses conceitos e metodologia em experimentos especialmente planejados para o estudo da eficiência de algoritmos para modelos de redes, como em Marins et al. (1997).

Neste trabalho, tratou-se de uma aplicação pouco comum, talvez pioneira, das idéias de Fisher na área gerencial. Não se pode negar, contudo, que foi com certo empirismo, mas com base na larga experiência da equipe de consultores da empresa estudada, que se determinaram os principais fatores que influenciam no tempo de implantação de sistemas ERP.

O objetivo, portanto, foi a melhoria da qualidade do serviço prestado por uma importante empresa fornecedora de aplicativos de sistemas ERP, facilitando o cumprimento de prazos e os cronogramas preestabelecidos. Verificouse que ambos os fatores analisados (ano de implantação do ERP e porte da empresa/cliente) possuem grande influência no tempo total de implantação desses sistemas, tornando possível a segmentação dos clientes em grupos distintos, seguindo os níveis dos fatores estudados. 
Tabela 3 - Resultado da aplicação do algoritmo do mínimo corte.

\begin{tabular}{|c|c|c|c|c|c|c|c|c|c|}
\hline \multirow{2}{*}{ Iteração k } & \multirow{2}{*}{$\begin{array}{l}\text { Atividades } \\
\text { grupo (a) }\end{array}$} & \multirow{2}{*}{$\begin{array}{l}\text { Atividade } \\
\text { escolhida }\end{array}$} & \multicolumn{6}{|c|}{ Duração das atividades } & \multirow{2}{*}{$\begin{array}{l}\text { Caminhos da rede de fluxo } \\
\text { [duração] }\end{array}$} \\
\hline & & & $\mathbf{A}$ & B & $\mathbf{C}$ & D & $\mathbf{E}$ & $\mathbf{F}$ & \\
\hline Início & $\mathrm{B}, \mathrm{C}, \mathrm{D}, \mathrm{E}$ & - & 4 & 10 & 6 & 12 & 6 & 4 & $\begin{array}{l}(\mathrm{BCDF})[32] \\
(\mathrm{BCEF})[26]\end{array}$ \\
\hline 1 & $\mathrm{~B}, \mathrm{C}, \mathrm{D}, \mathrm{E}$ & B & & 9 & & & & & $\begin{array}{l}\text { (BCDF) [31] } \\
\text { (BCEF) [25] }\end{array}$ \\
\hline 2 & B, C, D & B & & 8 & & & & & $\begin{array}{l}\text { (BCDF) [30] } \\
(\mathrm{BCEF})[24]\end{array}$ \\
\hline 3 & $\mathrm{~B}, \mathrm{C}, \mathrm{D}$ & B & & 7 & & & & & $(\mathrm{BCDF})[29]$ \\
\hline 4 & $\mathrm{C}, \mathrm{D}$ & $\mathrm{C}$ & & 7 & 5 & & & & (BCDF) [28] \\
\hline 5 & C, D & $\mathrm{C}$ & & 7 & 4 & & & & $(\mathrm{BCDF})[27]$ \\
\hline 6 & $\mathrm{D}$ & $\mathrm{D}$ & & 7 & 4 & 11 & & & $(\mathrm{BCDF})[26]$ \\
\hline 7 & $\mathrm{D}$ & $\mathrm{D}$ & & 7 & 4 & 10 & & & (BCDF) [25] \\
\hline 8 & $\mathrm{D}$ & $\mathrm{D}$ & 4 & 7 & 4 & 9 & 6 & 4 & $(\mathrm{BCDF})[24]$ \\
\hline
\end{tabular}

Essa classificação facilita o acesso às informações cadastradas em históricos na medida em que é possível realizar comparações ou até benchmarking entre empresas nas mesmas condições. Por exemplo, para afirmar que é possível implementar o ERP em seis meses, é necessário conhecer as condições do projeto, se a empresa é de pequeno ou médio porte. Da mesma forma, não se pode estimar que serão necessários ao menos dois anos para a implantação. Essa variável, tempo de implantação do sistema, depende de alguns fatores, que analisados no presente trabalho esclarecem e facilitam definições de prazos.

Antes deste trabalho, a determinação da duração de um projeto para a empresa estudada era realizada por meio de empirismo e da analogia com outras empresas e outros projetos similares. Conhecendo-se a classificação das empresas, a determinação dos prazos adquire maior confiabilidade. O estudo sobre a influência de fatores permite, agora, melhor planejamento sobre a duração total do projeto.
Entretanto, a determinação de prazos com maior confiabilidade não é suficiente para melhorar a qualidade da empresa. Para completar o estudo foram utilizadas técnicas de gerenciamento de projetos que permitem o controle sobre o prazo definido ao longo de cada atividade.

Assim, a técnica PERT permitiu o planejamento e o controle de todo o projeto, assim como encontrar as atividades críticas, facilitando o gerenciamento dos recursos ao longo do projeto. Com os resultados obtidos pela aplicação dos algoritmos detectou-se que algumas atividades críticas são o desenho da solução e a análise operacional. Esse resultado facilita o gerenciamento do projeto, pois indica em que fases são necessários maior controle e investimentos e quais outras atividades possuem alguma folga.

\section{Agradecimentos}

Aos revisores da revista Gestão \& Produção por suas sugestões para a melhoria do artigo.

\section{ANEXO - Metodologia AIM (application implementation method)}

A metodologia AIM foi estruturada para tornar ágil a implementação de sistemas ERP e é composta pelas seguintes fases: definição, análise operacional, desenho da solução, construção, transição e produção. Breve comentário de cada uma dessas fases:

(a) Definição: é a fase de planejamento da implementação do ERP, em que são identificados os requerimentos do negócio e dos sistemas de informação. São definidas a estratégia de implementação e as necessidades de arquitetura técnica e da aplicação de acordo com o modelo de negócio requerido. (b) Análise operacional: feita quando são levantadas as necessidades e informações relativas aos processos de negócio e em termos de tecnologia. A equipe de projeto cria um modelo para a estrutura da aplicação e sugere a arquitetura técnica geral.

(c) Desenho da solução: objetiva-se criar uma solução otimizada em termos de processos de negócio atendendo às necessidades futuras de negócio da empresa de manutenção. Criam-se narrativas detalhadas de processos determinados pela associação de características do ERP às necessidades do negócio identificados na fase anterior. 
(d) Construção: são feitas as codificações e testes dos programas.

(e) Transição: implementa-se a aplicação em seu ambiente operacional. $\mathrm{O}$ início está condicionado à plena conclusão da etapa anterior. (f) Produção: é o último estágio de implementação do ERP e o primeiro do ciclo de suporte de sistemas em produção, em que se incluem refinamentos e medições. A equipe de tecnologia da informação da empresa de manutenção trabalhará para promover a estabilização do sistema e começar sua manutenção regular.

\section{Referências Bibliográficas}

CAMPOS, V. F. Gerenciamento pela rotina do trabalho do dia-a-dia. Belo Horizonte: Editora DG, 1995.

CONTADOR, J.L. Algoritmo da folga mínima para determinação do caminho K-crítico em redes PERT. In: SIMPÓSIO BRASILEIRO DE PESQUISA OPERACIONAL. Anais... Campos do Jordão, SP, nov. 2001.

CONTADOR, J. L. Algoritmo do corte mínimo para aceleração de projetos. In: ENCONTRO NACIONAL DE ENGENHARIA DE PRODUÇÃO. Anais... Curitiba, PA, out. 2002.

CONTADOR, J. L. Gerenciamento de projetos com PERT/ CPM. In: Gestão de operações. São Paulo: Editora Edgard Blücher, 1997.

COSTA NETO, P. L. O. Estatística. 2. ed. São Paulo: Editora Edgard Blücher, 2001.

FISHER, R. A . The design of experiments. 8. ed. New York: Hafner Publishing Company, 1966.
GOMES, C.; VANALLE, R. Aspectos críticos para a implantação de sistemas ERP. In: ENCONTRO NACIONAL DA ENGENHARIA DE PRODUÇÃO. Anais... Salvador, 2001.

KOCH, C.; SLATER, D.; BAATZ, E. The ABCs of ERP. Disponível em: <http: //www.cio.com>. Acesso em: 22 ago. 2001.

MARINS, F. A . S.; SENNE, E. L. F.; MACHADO, A .; PERIN, C.; DARBYDOWMAN, K. Algorithms for Network Piecewise-Linear Programs: a comparative study. European Journal of Operational Research, v. 1, p. 183-199, 1997.

MONTGOMERY, D. C. Design and analysis of experiments. 4. ed. John Wiley \& Sons, 2001.

PRADO, D. PERT/CPM. Belo Horizonte: Editora DG, 1998.

STRAUSS, R. Managing multimedia projects. USA: Butterworth-Heinemann, 1997.

TAGUCHI, G. et al. Engenharia da qualidade em sistemas de produção. São Paulo: McGraw-Hill, 1989.

\section{ERP SYSTEMS INTRODUCTION TIME: FACTORS, ANALYSIS AND APPLICATION OF PROJECTS MANAGEMENT TECHNIQUES}

\section{Abstract}

Usually the implementation of ERP systems is very complex, and not immediate, in some cases requires three or even four years. In general, the ERP system is divided in modules, and its implementation is made in stages. At each stage, a few modules are implemented. According to several authors the deadlines of modules implementation are critical, and rarely are obeyed, so such delays make the customer insatisfied because they result in additional costs for them. The implementation of ERP are dictaded by several factors, some of them have more influence on its duration. The technique of Design Planning is considered here to determine these factors. Moreover, using Critical Path Methods are identified the bottleneck activities which require more investment in order to reduce the duration of whole project.

Key words: ERP systems introduction, design of experiments, critical path methods. 